\title{
Intermittent cafeteria diet identifies fecal microbiome changes as a predictor of spatial recognition memory impairment in female rats
}

\author{
Sarah-Jane Leigh', Nadeem O. Kaakoush', Michael J. Bertoldo², R. Frederick Westbrook ${ }^{3}$ and Margaret J. Morris ${ }^{1}$
}

\begin{abstract}
Excessive consumption of diets high in saturated fat and sugar impairs short-term spatial recognition memory in both humans and rodents. Several studies have identified associations between the observed behavioral phenotype and diet-induced changes in adiposity, hippocampal gene expression of inflammatory and blood-brain barrier-related markers, and gut microbiome composition. However, the causal role of such variables in producing cognitive impairments remains unclear. As intermittent cafeteria diet access produces an intermediate phenotype, we contrasted continuous and intermittent diet access to identify specific changes in hippocampal gene expression and microbial species that underlie the cognitive impairment observed in rats fed continuous cafeteria diet. Female adult rats were fed either regular chow, continuous cafeteria diet, or intermittent cafeteria diet cycles (4 days regular chow and 3 days cafeteria) for 7 weeks (12 rats per group). Any cafeteria diet exposure affected metabolic health, hippocampal gene expression, and gut microbiota, but only continuous access impaired short-term spatial recognition memory. Multiple regression identified an operational taxonomic unit, from species Muribaculum intestinale, as a significant predictor of performance in the novel place recognition task. Thus, contrasting intermittent and continuous cafeteria diet exposure allowed us to identify specific changes in microbial species abundance and growth as potential underlying mechanisms relevant to diet-induced cognitive impairment.
\end{abstract}

\section{Introduction}

There is strong evidence that diets high in saturated fat and refined carbohydrates are associated with cognitive impairments in humans ${ }^{1}$ and rodents ${ }^{2,3}$, but the mechanisms underlying this association remain unclear. Such diets compromise physiological functions across multiple organ systems, many of which could contribute to the observed cognitive impairments. Therefore, identifying key physiological disruptions that contribute to diet-induced cognitive dysfunction is essential to better understand and mitigate the effects of poor diet and obesity on cognition.

\footnotetext{
Correspondence: Margaret J. Morris (m.morris@unsw.edu.au)

'School of Medical Sciences, UNSW, Sydney, NSW 2052, Australia

'Fertility and Research Centre, School of Women's and Children's Health, UNSW, Sydney, NSW 2052, Australia

Full list of author information is available at the end of the article.
}

We and others have used rodent models to show that a western-style cafeteria diet, high in fat and sugar, promotes prolonged hyperphagia and increases adiposity, compromising metabolic health ${ }^{4-6}$. Exposure to such diets impairs performance on hippocampal-dependent tasks assessing short-term spatial recognition ${ }^{2,7}$ and reference ${ }^{8,9}$ memory, as well as disrupting multiple hippocampal molecular pathways, including pro-inflammatory signaling ${ }^{7,8,10}$, blood-brain barrier integrity ${ }^{3,11}$ and synaptic transmis$\operatorname{sion}^{12}$, and inducing fecal microbiome perturbations ${ }^{13,14}$. However, no one of these physiological changes has been consistently tied to the behavioral phenotype and limited studies targeting causation have been undertaken.

We have also shown that intermittent access to a western-style cafeteria diet (3 consecutive days of cafeteria diet followed by 4 consecutive days of chow diet each week for 16 weeks) resulted in similar fecal 
microbiome perturbations to those observed in rats continuously consuming cafeteria diet $^{15}$ with intermediate alterations to metabolic health ${ }^{16}$. Of particular interest, such intermittent exposure to cafeteria diet does not impair spatial memory task performance compared with young male rats maintained on a healthy diet ${ }^{17}$, while still impairing metabolic health. This intermediate phenotype provides an opportunity to identify the key changes in hippocampal function and microbiome composition that are associated with diet-induced cognitive impairment, separate from more general changes produced by a cafeteria diet.

The present study aimed to determine whether specific differences in hippocampal gene expression and microbiome composition between intermittent (Cycle) and continuous cafeteria (Cafeteria) diet exposure contributed to the severity of spatial memory impairment. We chose to investigate these differences in females, as there are limited preclinical studies of the effects of obesogenic diets on cognition in female rodents despite a higher incidence of weight cycling ${ }^{18}$ and dieting ${ }^{19}$ in women.

\section{Materials and methods}

\section{Ethics statement}

This protocol was approved by the Animal Care and Ethics Committee of UNSW Sydney in accordance with the Australian guidelines for the use and care of animals for scientific purposes (Australian National Health and Medical Research Council).

\section{Subjects and diet manipulation}

Thirty-six female Sprague-Dawley rats aged 4-5 months (Animal Resource Centre, Australia) were housed three per box $\left(18-22^{\circ} \mathrm{C} ; 12 \mathrm{~h}\right.$ light/dark). Rats were handled daily for 1 week, while maintained on standard chow $(11 \mathrm{~kJ} / \mathrm{g}$; Premium Rat and Mouse Maintenance diet; Gordon's Specialty Stockfeeds, Australia) and water ad libitum.

Following acclimatization, weight-matched groups were randomly allocated to receive Chow, Cycle, or Cafeteria diet ( $n=12$ rats, $n=4$ cages per group) ad libitum. The sample size was based on previous power calculations showing a minimum of ten rats is needed to detect hippocampal gene expression differences with a moderate effect size. Chow rats remained on standard chow and water. Cycle rats were exposed to 3 consecutive days of cafeteria diet, then 4 consecutive days of Chow for 7 weeks. Cafeteria rats had continuous access to cafeteria diet (full protocol in ref. ${ }^{20}$ ) consisting of standard chow, water, and $10 \%$ sucrose solution, alongside commercially produced cakes (e.g., mud cake and jam roll), biscuits (e.g., choc-chip cookies and custard creams), and savory foods (e.g., meat pie and dim sims).
Body weight and food intake were measured twice weekly. For food intake, food items were weighed before administration and reweighed following $24 \mathrm{~h}$ after carefully removing all food from the cage. The amount eaten and drunk per home cage was converted to $\mathrm{kJ}$ using data provided by the manufacturers and the average intake per rat was calculated assuming equal intake in a cage. Body composition was analyzed at the end of week 7 by EchoMRI-900 (EchoMRI LLC, USA).

\section{Behavior: novel object and novel place tasks}

Hippocampal-dependent spatial and perirhinal-dependent object recognition memory were assessed using the novel place recognition task (NPR) at 3 and 6 weeks, and novel object recognition task (NOR) at 4 weeks.

The apparatus was a square arena $(60 \mathrm{~cm} \times 60 \mathrm{~cm} \times$ $60 \mathrm{~cm}, 40$ lux). For the NOR, objects were matched on volume and color, but differed in shape and material. Object type and locations were counterbalanced across treatment groups and across time. No object was used twice for any rat. Videos were recorded to mask experimental group prior to scoring.

Two days prior to the first NPR, rats were exposed to the empty arena for $10 \mathrm{~min}$ each day. Both NPR and NOR tasks consisted of familiarization, retention, and test phases. During familiarization, rats were placed into the arena with two identical novel objects and allowed to explore for $5 \mathrm{~min}$. They were then returned to their home cage for a $5 \mathrm{~min}$ retention period, while the arena and objects were cleaned with $40 \%$ ethanol.

Immediately following retention, animals underwent a 3 min test. For NOR, objects were placed in the same locations as familiarization, with one novel object and one object identical to those used previously. In NPR, the objects were identical to those shown previously, but one object was moved to a new location, while the other remained in the original location. Exploration ratio was calculated as (novel exploration time) $/($ novel + familiar exploration time).

\section{Estrous cycle monitoring}

As this study examined females and diet is known to affect both the estrous cycle ${ }^{21,22}$ and oocyte health ${ }^{23}$, and there is mixed evidence for a moderating role of estrous cycle in spatial cognition ${ }^{24,25}$, diet- and microbiomeassociated differences in estrous cycle and oocyte number were investigated. During weeks $1-2$ and 6-7, daily estrous monitoring was performed by vaginal lavage. Epithelial changes were stained using 0.1\% toluidine blue stain and were classified as previously reported ${ }^{26}$.

\section{Sample collection}

Following 7 weeks, rats were deeply anesthetized (ketamine/xylazine 15/100 mg/kg intraperitoneally). All 
rats had access to their respective diets until killing (Cycle on cafeteria diet). Body weight, naso-anal length, girth, and blood glucose were measured following induction of anesthesia. Blood was obtained by cardiac puncture and rats were decapitated. Plasma was stored at $-80^{\circ} \mathrm{C}$ for subsequent determination of leptin, insulin, and triglyceride content.

The dorsal hippocampus (within a coronal block defined by the rostro-caudal limits of the Circle of Willis) was rapidly dissected and collected. Retroperitoneal white adipose tissue and liver were dissected and weighed. One fecal pellet was removed from the distal colon. Hippocampus and feces were snap frozen in liquid nitrogen and stored at $-80{ }^{\circ} \mathrm{C}$ for analysis. Ovaries were dissected and oocytes recovered and counted.

\section{Plasma hormone and triglyceride measurements}

Plasma leptin and insulin concentrations were obtained using commercial kits according to manufacturer's instructions (CAT\#90040 and CAT\#90060, CrystalChem, Inc., USA).

Plasma triglyceride content was measured spectrophotometrically using triglyceride reagent (Roche Diagnostics Australia Pty, Ltd, Australia) at $37^{\circ} \mathrm{C}$ alongside a standard curve generated from glycerol standard (G77935ML, Sigma-Aldrich Pty, Ltd, Australia).

\section{Hippocampal gene expression}

RNA was extracted from dorsal hippocampus using a TRI Reagent protocol (Sigma-Aldrich Pty, Ltd, Australia). Following DNAse I treatment (Catalog\# 42885; Merck, Australia), $1.5 \mu \mathrm{g}$ of RNA were reverse transcribed to produce cDNA (High Capacity Reverse Transcriptase Kit; Thermo Fisher Scientific, USA). Gene expression was assessed using Taqman inventoried gene expression assays (Life Technologies Australia Pty, Ltd, Australia; details in Supplementary Table 2). Genes of interest were normalized against the geometric mean of the two most stable housekeeping genes (Ywhaz and Hprt1) identified by Normfinder code in $R^{27}$. Analysis of relative gene expression was performed using the $\Delta \Delta C T$ method normalized to an independent calibrator ${ }^{28}$.

\section{Statistical analyses}

Data were analyzed using one-way analysis of variance (ANOVA) or mixed two-way ANOVA (for measures over time) with post-hoc Tukey's comparisons. Where data violated homoscedasticity of variance, $\log$ transformations were employed. Ordinal variables were analyzed using an independent-samples Jonckheere-Terpstra test for ordered alternatives followed by non-parametric Bonferroni-Dunn post-hoc testing. All post-hoc comparisons are presented only in the associated figures and tables when $p<0.05$. Pearson's correlations were performed to identify key variables of interest associated with NPR performance, which was followed up using simultaneous multiple regression to identify key predictors. All analyses were completed using IBM SPSS Statistics 23 (Australia). One Cafeteria rat was excluded due to poor health (hepatomegaly).

\section{Fecal DNA extraction, microbiome community sequencing, and statistical analyses}

DNA extraction was performed using the PowerFecal DNA Isolation Kit (MoBio Laboratories, USA). Microbial community composition was assessed by Illumina amplicon sequencing $(2 \times 250 \mathrm{bp}$ MiSeq chemistry, V4 region, 515F-806R primer pair) using a standard protocol. Sequence data were analyzed using MOTHUR ${ }^{29}$, using modified commands from MiSeq $\mathrm{SOP}^{30}$, including alignment with the SILVA database, singleton removal, chimera checking with UCHIME, and classification against the latest RDP training set. Sequence data $(n=8346$ total clean reads/sample) did not undergo rarefaction.

Operational taxonomic unit (OTU) correlations and LefSe analyses were completed using Calypso ${ }^{31}$, where multiple testing was corrected using the Benjamini-Hochberg false discovery rate (FDR). Alpha diversity metrics were obtained from Calypso and analyses were completed using SPSS, whereas FDR-corrected DESeq2 was performed using $\mathrm{R}$, using the Phylose $\mathrm{q}^{32}$ package for the negative binomial Walk's test in DESeq2 ${ }^{33}$. OTU abundances were analyzed using SPSS with Kruskal-Wallis tests when necessary, followed by non-parametric Bonferroni-Dunn post-hoc testing where appropriate. OTUs of interest were identified using SINA Aligner ${ }^{34}$ and BlastN.

Distanced-based linear modeling (dbLM), permutational ANOVA (PERMANOVA), non-metric multidimensional scaling (nMDS), and canonical analysis of principal coordinates were completed using Primer (Primer-E Ltd, Plymouth, $\mathrm{UK}^{35}$ ). All primer analyses utilized a Bray-Curtis similarity matrix constructed at the OTU level. Marginal dbLM interrogates the unique contribution of each predictor variable to the variance in the Bray-Curtis similarity matrix.

Phylogenetic Investigation of Communities by Reconstruction of Unobserved States (PICRUSt) was performed using Galaxy web, to predict putative functions (through metagenomic prediction) from the 16S OTU data using Greengenes 13.5 for taxonomic classification $^{36}$. Pathway counts were compared across groups using FDR-corrected Kruskal-Wallis tests followed by non-parametric Bonferroni-Dunn post-hoc testing, where appropriate. 


\section{Results}

Continuous cafeteria diet impairs metabolic health more than diet cycling

Access to cafeteria diet consistently elevated daily energy intake: Cafeteria and Cycle rats consumed on average 3.8- and 1.8-fold more energy than chow controls $(41,920 \mathrm{~kJ} / \mathrm{rat}, 19,980 \mathrm{~kJ} / \mathrm{rat}$, and $11,133 \mathrm{~kJ} / \mathrm{rat}$ total energy, respectively). Based on food-intake measurements, Cafeteria rats consumed 58\% carbohydrates, $8 \%$ protein, and $34 \%$ fat as energy; similarly, Cycle rats consumed $56 \%$ carbohydrates, $8 \%$ protein, and $35 \%$ fat while on cafeteria diet. Chow diet comprised $65 \%$ carbohydrate, $22 \%$ protein, and $13 \%$ fat.

Absolute protein intake was slightly elevated in Cafeteria rats relative to Chow. Cycle rats ate comparable protein to Cafeteria rats, while consuming cafeteria diet; however, they consumed slightly less protein than Chow rats overall (Supplementary Fig. 1C; total protein: Chow: $2473 \mathrm{~kJ} / \mathrm{rat}$, Cycle: $2036 \mathrm{~kJ} / \mathrm{rat}$, and Cafeteria: $3092 \mathrm{~kJ} / \mathrm{rat}$ ). The intake of carbohydrate and fat (Supplementary Fig. 1D and E) when Cycle rats were exposed to the cafeteria diet were comparable to those by Cafeteria rats and were higher than Chow intakes when summed over the study (total carbohydrate: Chow: $7418 \mathrm{~kJ} /$ rat, Cycle: $11,524 \mathrm{~kJ} /$ rat, Cafeteria: 24,351 kJ/rat; total fat: Chow: $1538 \mathrm{~kJ} / \mathrm{rat}$, Cycle: $6192 \mathrm{~kJ} /$ rat, Cafeteria: 14,184 kJ/rat).
Body weight gain over the study was significantly different between groups $(F(2,32)=49.62, p<0.001$; Supplementary Fig. 1F for body weight over time) such that weight gain was greater in Cafeteria rats than Chow and Cycle rats. There was no significant difference between Chow and Cycle rats, in line with our previous work in male rats ${ }^{37}$.

Continuous cafeteria diet increased body weight, fat mass, liver weight, and blood glucose, as well as plasma insulin, leptin, and triglyceride concentrations, relative to Chow rats with less marked effects in Cycle rats. Although lean mass did not differ, fat mass and leptin concentrations were significantly different among all groups, increasing with diet exposure. Oocyte number was reduced in Cafeteria rats relative to Chow controls (Table 1).

\section{Intermittent cafeteria diet spares cognition and increases the expression of some hippocampal pro-inflammatory genes}

Short-term spatial and object memory recognition were assessed using NPR and NOR tasks, respectively; when assessed with a short retention period ( $5 \mathrm{~min}$ ), the NPR is predominantly hippocampal-dependent, whereas the NOR relies on an intact perirhinal cortex ${ }^{38,39}$. Previously, we have shown that NPR, but not NOR, is impaired by exposure to cafeteria diet in rats ${ }^{2,7}$.

Table 1 Body composition, tissue collection, and plasma measures.

\begin{tabular}{|c|c|c|c|c|}
\hline & Chow & Cycle & Cafeteria & Diet effect \\
\hline \multicolumn{5}{|l|}{ EchoMRI (3 days prior to kill) } \\
\hline Fat mass (g) & $46.8 \pm 4.1$ & $73.1 \pm 6.8^{\mathrm{a}}$ & $126.1 \pm 9.6^{a, b}$ & $p<0.001$ \\
\hline Fat percentage (\%) & $14.2 \pm 1.1$ & $20.7 \pm 1.4^{\mathrm{a}}$ & $29.5 \pm 1.4^{a, b}$ & $p<0.001$ \\
\hline Lean mass (g) & $262.6 \pm 6.5$ & $256.2 \pm 5.4$ & $277.0 \pm 7.4$ & - \\
\hline \multicolumn{5}{|l|}{ Tissue collection } \\
\hline Final body weight (g) & $329.9 \pm 7.9$ & $367.0 \pm 12.0$ & $433.4 \pm 17.3^{a, b}$ & $p<0.001$ \\
\hline Liver weight (g) & $9.95 \pm 0.35$ & $11.96 \pm 0.54^{\mathrm{a}}$ & $12.68 \pm 0.49^{\mathrm{a}}$ & $p=0.001$ \\
\hline $\begin{array}{l}\text { Retroperitoneal fat pad } \\
\text { weight }(\mathrm{g})\end{array}$ & $4.81 \pm 0.45$ & $9.40 \pm 1.14^{\mathrm{a}}$ & $13.15 \pm 0.71^{a, b}$ & $p<0.001$ \\
\hline Ovary weight (mg) & $46.0 \pm 2.1$ & $44.7 \pm 1.93$ & $50.2 \pm 2.4$ & - \\
\hline Total oocytes/female & $20.5 \pm 1.3$ & $17.1 \pm 1.5$ & $15.1 \pm 1.8^{\mathrm{a}}$ & $p=0.024$ \\
\hline \multicolumn{5}{|l|}{ Blood/plasma measures } \\
\hline Blood glucose (mmol/L) & $7.7 \pm 0.3$ & $8.3 \pm 0.3$ & $9.3 \pm 0.5^{\mathrm{a}}$ & $p=0.014$ \\
\hline Plasma insulin (ng/mL) & $0.19 \pm 0.05$ & $0.91 \pm 0.20^{\mathrm{a}}$ & $1.08 \pm 0.22^{\mathrm{a}}$ & $p=0.002$ \\
\hline Plasma leptin (ng/mL) & $5.69 \pm 0.68$ & $11.35 \pm 1.28^{\mathrm{a}}$ & $17.38 \pm 1.67^{\mathrm{a}, \mathrm{b}}$ & $p<0.001$ \\
\hline Plasma triglycerides (mmol/L) & $1.06 \pm 0.08$ & $2.30 \pm 0.34^{a}$ & $3.48 \pm 0.49^{\mathrm{a}}$ & $p=0.001$ \\
\hline
\end{tabular}

Results expressed as mean \pm SEM; $n=11-12$; data were analyzed by one-way ANOVA followed by Tukey-adjusted post-hoc testing $\left({ }^{\mathrm{a}} p<0.05\right.$ relative to Chow, ${ }^{\mathrm{b}} p<$ 0.05 relative to $\mathrm{Cycle}$ ). Total oocytes per female were analyzed using an independent-samples Jonckheere-Terpstra test for ordered alternatives followed by nonparametric Bonferroni-Dunn post-hoc testing $\left({ }^{\mathrm{a}} p<0.05\right.$ relative to Chow). 
Following 3 weeks of diet exposure, NPR memory was significantly affected by diet $(\mathrm{F}(2,32)=9.08, p<0.001$; Fig. $1 \mathrm{~b}$ and Supplementary Fig. 2A, B). Although both Chow and Cycle rats performed significantly better than Cafeteria, there was no difference between Chow and Cycle rats. By contrast, there were no significant group differences for NOR at 4 weeks $(\mathrm{F}(2,32)<1$; Supplementary Fig. $2 \mathrm{E}, \mathrm{F})$, consistent with previous work $^{2,7}$.

The group differences in NPR performance present at 3 weeks remained significant at 6 weeks $(F(2,32)=7.57$, $p=0.022$; Fig. 1a, b), when Cycle rats had experienced 19-20 days total cafeteria diet, matching exposure of Cafeteria rats at the 3 -week time point. Tested 4 days later, although Cycle rats were consuming chow, the overall effect of diet remained significant $(\mathrm{F}(2,31)=5.71$, $p=0.008$; Supplementary Fig. 2C, D) such that Cafeteria rats were significantly impaired relative to Cycle rats. There was no significant difference between Chow and Cycle rat performance on the NPR task. Exploration times were not significantly different between groups for any of the tasks (Supplementary Fig. 1).

As there is some evidence that spatial cognition may vary with estrous cycle in female rodents ${ }^{24,25}$, we assessed estrous cycle after the final NPR. No relationship was observed with NPR performance, although this was not explicitly manipulated, as we did not want to introduce variability in dietary exposure by matching estrous cycle across groups.

Hippocampal gene expression was assessed to determine whether diet affected markers of pro-inflammatory signaling, blood-brain barrier integrity, and synaptic function (Fig. 1c-e and Supplementary Table 1 for gene names). Overall, these results indicate that although any exposure to cafeteria diet increases hippocampal cytokine expression, increased markers of astroglial and microglial proliferation, downstream pro-inflammatory signaling, and changes to blood-brain barrier integrity were unique to continuous cafeteria diet exposure. Specifically, any cafeteria diet exposure increased expression of $I l 1 b$ (F $(2,29)=6.13, p=0.006)$, Il6 $(\mathrm{F}(2,29)=5.17, p=0.012)$, Ikbkb $(\mathrm{F}(2,29)=17.05, p<0.001)$, and Drd2 $(\mathrm{F}(2,29)=$ $6.091, p=0.006)$, while continuous access uniquely elevated expression of Gfap $(\mathrm{F}(2,29)=4.948, p=0.014)$, Glut1 $(\mathrm{F}(2,29)=3.827, p=0.036), C \ln 5(\mathrm{~F}(2,29=3.846$, $p=0.033)$, and Htr1a $(\mathrm{F}(2,29)=6.64, p=0.004)$ relative to Chow. Aif1 (gene for Iba1, a marker of microglial proliferation; $\mathrm{F}(2,29)=8.33, p=0.001)$ was significantly elevated in Cafeteria rats relative to both Chow and Cycle groups. Interestingly, Syn1 $(\mathrm{F}(2,29)=3.886, p=0.032)$, Mtor $(\mathrm{F}(2,29)=4.214, p=0.025)$, and $\operatorname{Mc} 4 r(\mathrm{~F}(2,29)=$ 5.98, $p=0.007$; Supplementary Fig. 3C) were elevated in Cycle rats, suggesting differences in synaptic plasticity and energy homeostasis signaling exclusively in this group. Lepr $(\mathrm{F}(2,29)=1.83$, 0.18; Supplementary Fig. 3A), Insr (F
$(2,29)=1.82, p=0.18$; Supplementary Fig. 3B), and Glut3 $(\mathrm{F}(2,29)=1.7, p=0.21$; Supplementary Fig. 3D) were also assessed to determine whether there were any group differences in metabolic signaling, but did not show statistically significant differences. Aif1 and Tlr2 were the only genes significantly associated with spatial recognition memory (Fig. If and g, and Supplementary Table 2), implying that bacterial lipoprotein content and microglial proliferation may be involved in diet-induced cognitive impairment.

\section{Intermittent cafeteria diet exposure does not affect a-diversity or predicted bacterial function, but alters microbiome composition}

Microbial species diversity was assessed using richness, evenness, and Shannon's diversity at 7 weeks. Both richness $(\mathrm{F}(2,33)=3.949, p=0.029$; Fig. 2a) and Shannon's diversity $(\mathrm{F}(2,33)=4.31, p=0.022$; Fig. $2 \mathrm{c})$ were significantly affected by diet overall, and the Cafeteria group showed reduced values relative to chow-fed controls. Evenness (Fig. 2b) was not significantly changed by diet. No significant effect of cage was observed.

PERMANOVA (999 permutations) at 7 weeks revealed significant differences in microbiome composition by diet (Pseudo-F $(2,24)=3.18, p=0.006$ ) and cage (Pseudo-F $(3,24)=1.51, p=0.001)$, and the nMDS plot illustrating the similarity between groups is presented in Fig. 2d. Canoncal analysis of principal coordinates revealed that although Cycle microbiome overlapped with both Chow and Cafeteria groups, it was more closely aligned with the Cafeteria microbiome composition: when combined Cycle and Cafeteria were compared with Chow, there was 0\% mis-classification error, whereas this was $14.3 \%$ for combined Cycle and Chow vs. Cafeteria. Overall, there were no significant differences in dispersion between groups ( $\mathrm{F}$ $(2,33)=1.55, p=0.235)$.

Several relative OTU abundances were selectively enriched in particular diet groups, using both LefSe and DeSeq2 analyses (DeSeq2: FDR-adjusted $p<0.05$; LefSe: LDA score $>2, p<0.05$; Fig. 2e). When comparing Chow and Cafeteria, 16 OTUs were enriched in cafeteria diet, whereas 15 OTUs were enriched in chow-fed controls, and similar differences were observed between Chow and Cycle rats. Between Cycle and Cafeteria groups, only two OTUs were differentially enriched: Porphyromonadaceae unclassified_OTU35 was enriched in Cycle rats, whereas probacter_OTU66 was relatively enriched in the Cafeteria group.

Predicted bacterial function was assessed using PICRUSt analysis of $16 \mathrm{~S}$ sequence data. Following FDR correction for multiple comparisons on relevant bacterial and metabolic KEGG (Kyoto Encyclopedia of Genes and Genomes) pathways, ether lipid metabolism $(\mathrm{H}(2)=$ 19.99, $p=0.005)$, flavone and flavonol biosynthesis 
A)

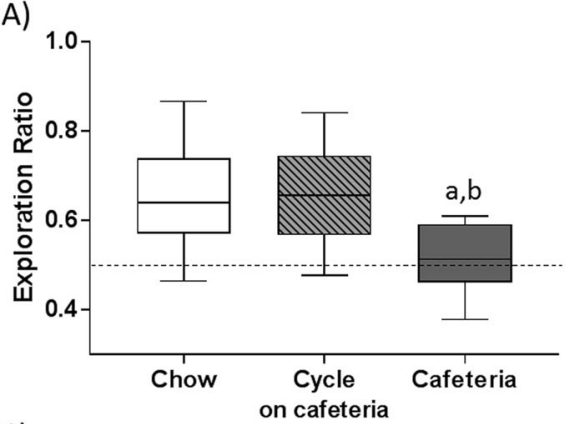

C)

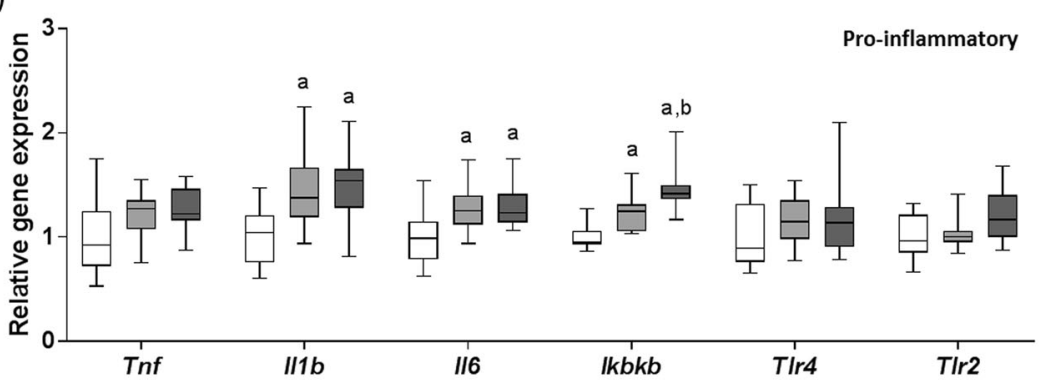

D)

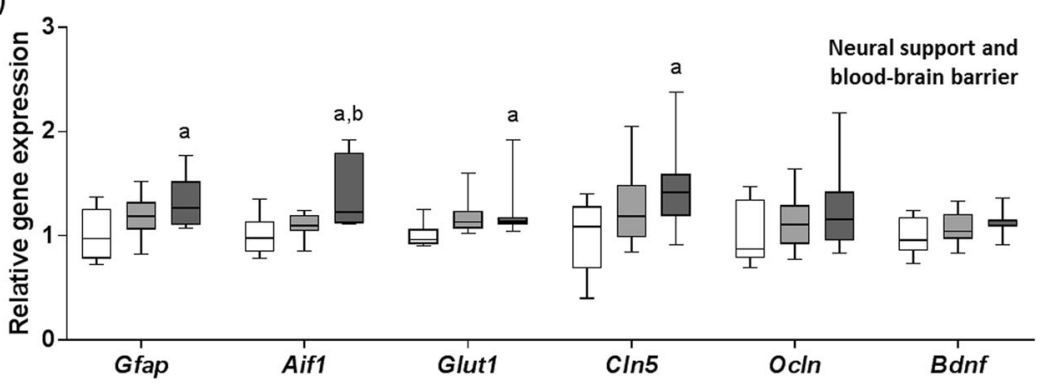

E)

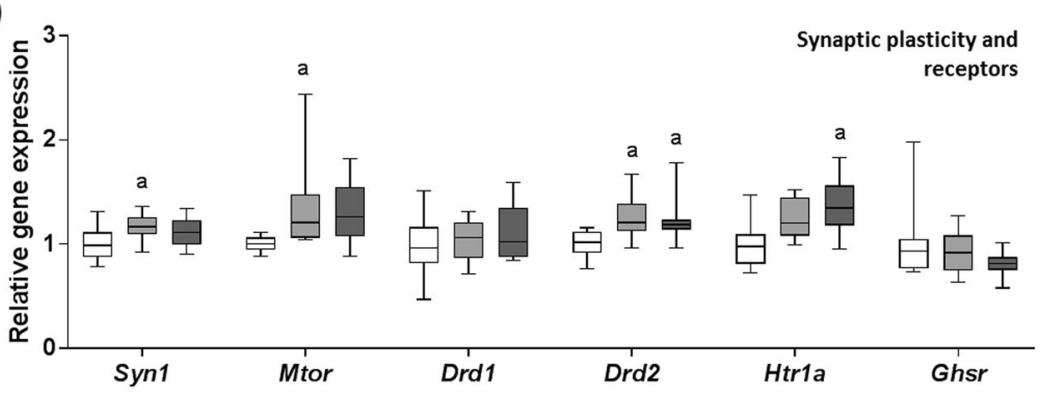

F)

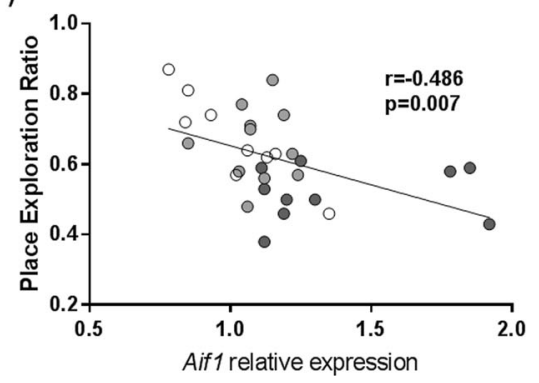

G)

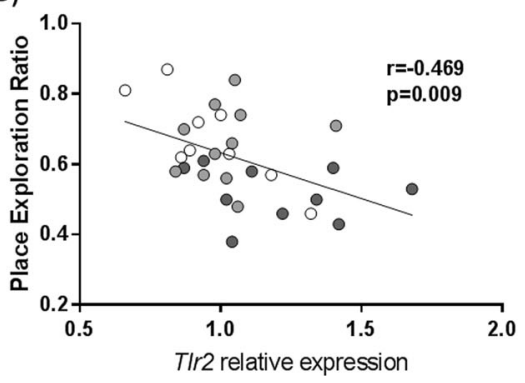

B)

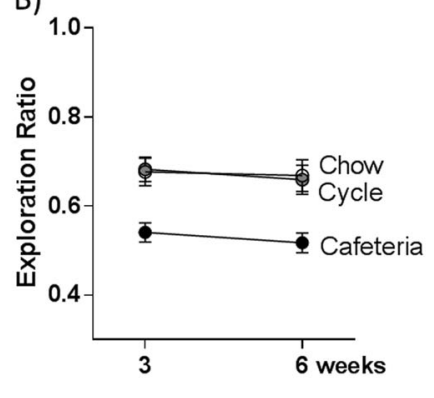

Fig. 1 (See legend on next page.) 
(see figure on previous page)

Fig. 1 Intermittent cafeteria diet exposure spares spatial recognition memory while increasing expression of some hippocampal proinflammatory genes. a Novel place recognition memory task performance at 6 weeks of diet exposure. b Novel place recognition memory task performance over the study. $\mathbf{c}$ Expression of pro-inflammatory genes in the dorsal hippocampus. $\mathbf{d}$ Expression of glial and blood-brain barrier-related genes in the dorsal hippocampus. e Expression of neuroplasticity-related and receptor genes in the dorsal hippocampus. Data are expressed as boxand-whisker plots (min, IQR, max); $n=10-12$; data were analyzed by one-way ANOVA followed by Tukey-adjusted post-hoc comparisons ( ${ }^{a} p<0.05$ relative to Chow, ${ }^{\mathrm{b}} p<0.05$ relative to Cycle). $\mathbf{f}$ Correlation between spatial recognition memory and hippocampal Aif1 gene expression. $\mathbf{g}$ Correlation between spatial recognition memory and hippocampal T/r2 gene expression. Data are expressed as scatterplots of individual values; $n=9-12 ;$ data were analyzed by Pearson's correlations. Aif1 allograft inflammatory factor 1, Bdnf brain-derived neurotrophic factor, Cln5 Claudin 5, Drd1 dopamine receptor D1, Drd2 dopamine receptor D2, Gfap glial fibrillary acidic protein, Ghsr growth hormone secretagogue receptor, Glut1 glucose transporter 1, Htrla serotonin receptor $1 \mathrm{a}$, Ikbkb inhibitor of nuclear factor $\mathrm{kB}$ kinase subunit $\beta, \| 16$ interleukin-1 $\beta$, 116 interleukin-6, Mtor mammalian target of rapamycin, Ocln occludin, Syn1 synapsin 1, T/r2 Toll-like receptor 2, T/r4 Toll-like receptor 4, Tnf tumor necrosis factor-a.

$(\mathrm{H}(2)=15.42, \quad p=0.005)$, and flavonoid biosynthesis $(\mathrm{H}(2)=16.90, p=0.001)$ were all significantly reduced by any cafeteria diet exposure but none of these changes were associated with NPR performance.

\section{Changes in global microbiome composition are associated with place task performance}

Several variables were significantly associated with overall microbiome composition when their unique variance was considered through dbLM (Table 2), including measures of adiposity as well as hippocampal $I k b k b$ and $I l 1 b$. Of note, NPR performance at 6 weeks was a significant predictor of global microbiome composition (Pseudo-F $=1.89, p=0.022, R^{2}=0.054$ ).

\section{Coprobacter_OTU66 and body length are significant predictors of place task performance}

Several variables of interest were significantly associated with spatial recognition memory using Pearson's correlations (Supplementary Table 2). Although many differentially abundant OTUs correlated with NPR performance, only the association with Coprobacter_OTU66 $(r=-0.456$, $p=0.008$; Fig. 3f) was not dependent on large outliers. Of note, this association was statistically significant in Chow rats only $(r=-0.899, p<0.001)$ when investigated within each diet group. Coprobacter_OTU66 was also significantly associated with girth, fat mass, and a number of hippocampal pro-inflammatory genes (Supplementary Table 2). These variables were then entered into a sequential multiple regression, to determine which significantly predicted NPR performance while accounting for other variables. The final model, presented in Fig. 3a, found that Coprobacter_OTU66 and naso-anal length significantly predict $41.4 \%$ of the variance in $\operatorname{NPR}(\mathrm{F}(2,30)=12.28, p<0.001)$. Naso-anal length did not differ significantly between groups (Fig. $3 \mathrm{~b}$ ), although its strong positive association with fat mass $(r=0.444, p=0.008$; Fig. 3d) and other measures of adiposity indicate that this may be an indirect measure of diet impact.

Coprobacter_OTU66 was enriched in Cafeteria rats relative to Chow, as well as in Cafeteria relative to Cycle rats (Fig. 3e), and positively associated with fat mass $(r=$ $0.379, p=0.025$; Fig. $3 g$ ). SINA Aligner analysis identified one uncultured bacterium from the family Muribaculaceae (Accession number EU451781 ENA) isolated from mouse cecum ${ }^{40}$ that shared $90.5 \%$ sequence identity with Coprobacter_OTU66. BLAST analysis showed Coprobacter_OTU66 shared 90\% sequence identify with Muribaculum intestinale strain YL27.

\section{Discussion}

In contrast to continuous access to cafeteria diet, we found that intermittent diet exposure in females (Cycle group) generated an intermediate phenotype, with moderate increases in energy intake and adiposity, perturbed fecal microbiome composition, but no cognitive impairment. This pattern of access to a cafeteria diet has been shown to spare spatial recognition in male rats ${ }^{17}$, which is interesting, as poor diet has been shown to impair cognition when presented in a daily limited access model $^{41}$ and when excess energy intake is prevented ${ }^{10}$. Such intermittent access to cafeteria may spare cognition through the hypophagia exhibited during access to healthy chow, as both chronic ${ }^{42}$ and intermittent ${ }^{43}$ caloric restriction have been shown to improve cognition in mice.

Although Cycle rats developed a similar metabolic phenotype to Cafeteria rats, they exhibited lower relative fat mass and plasma leptin concentrations, which could explain the apparent protective effects of intermittent cafeteria diet on cognition. Of note, the plasma measures were conducted while animals were unfasted, due to previous work showing that diet-induced hypothalamic neuroinflammation can be reversed with overnight fasting $^{44}$, and this could potentially mask differences in metabolic function between Cycle and Cafeteria rats. However, we have previously shown that diet-induced cognitive impairments occur without increased body weight $^{7,10}$. Furthermore, although changes in hippocampal pro-inflammatory gene expression (Tlr2 and Aif1) were associated with spatial recognition memory independently, as seen in other rodent studies ${ }^{2,11,45}$, these were no longer significant predictors of the behavioral 

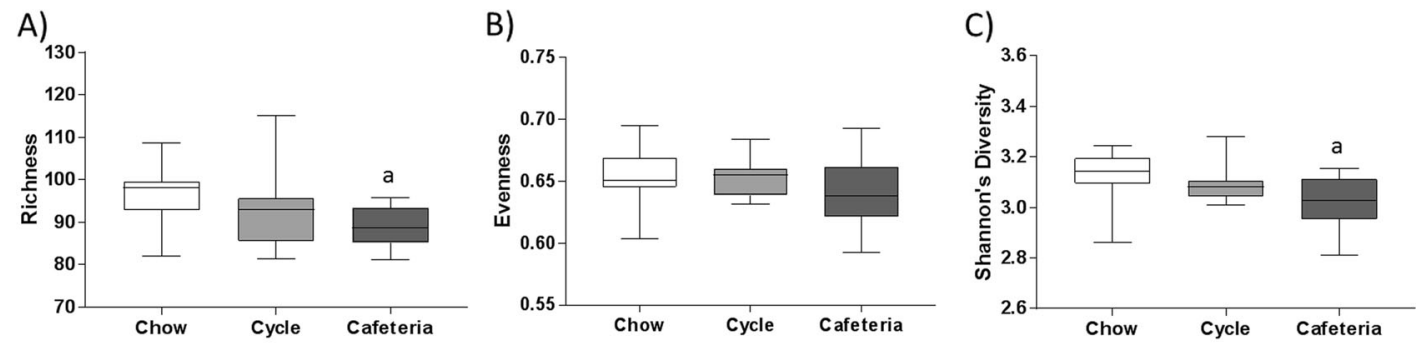

D)

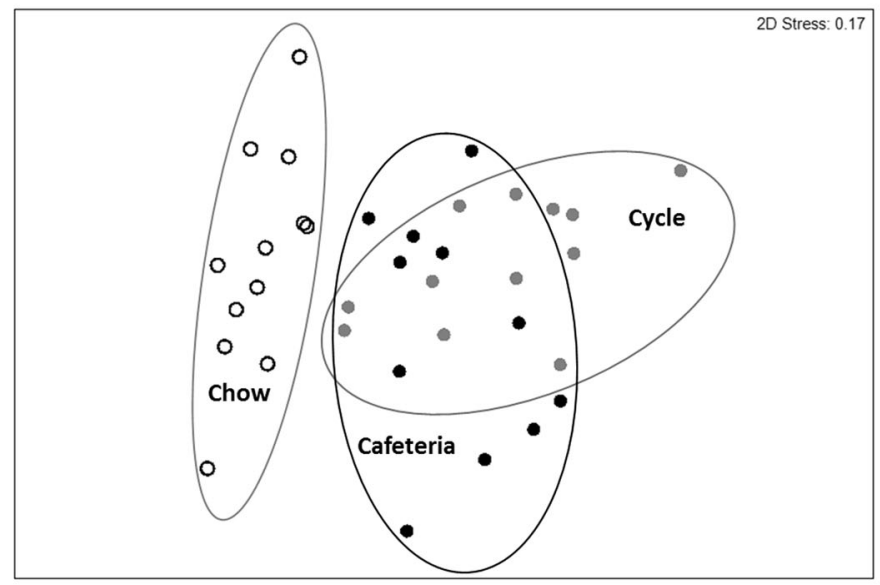

E)

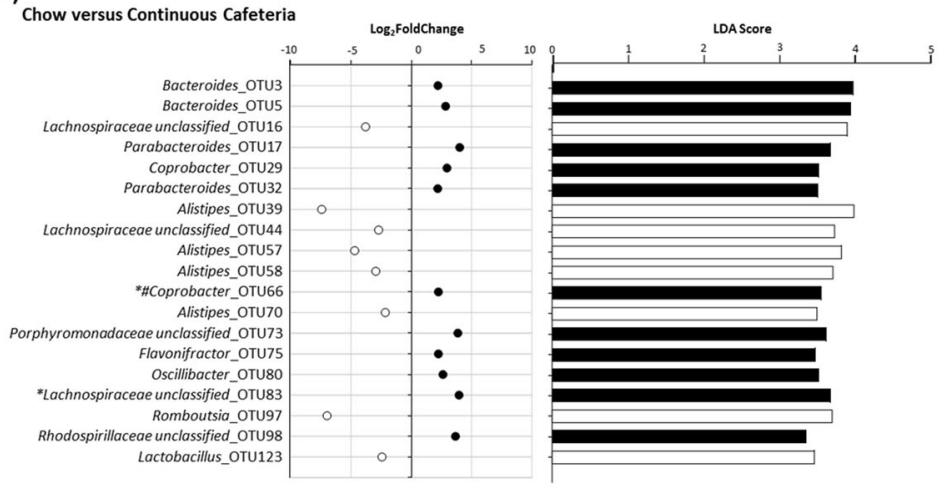

Cycling versus Continuous Cafeteria
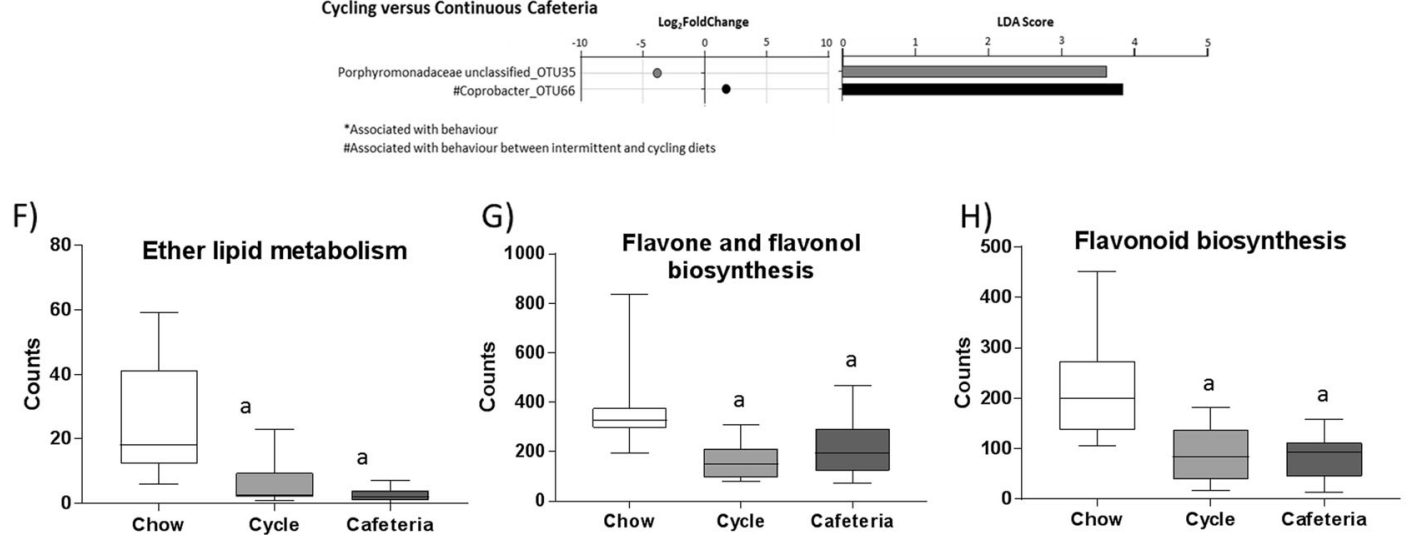

Fig. 2 (See legend on next page.) 
(see figure on previous page)

Fig. 2 Any cafeteria diet exposure affects gut microbiome composition and alters predicted bacterial metabolic pathways. a Microbial species richness, $\mathbf{b}$ microbial species evenness, and c Shannon's diversity following 7 weeks of diet exposure. Data are expressed as box-and-whisker plots (min, IQR, max); $n=11-12$; data were analyzed by one-way ANOVA followed by Tukey-adjusted post-hoc comparisons ( ${ }^{a} p<0.05$ relative to Chow). $\mathbf{d}$ Non-metric multidimensional scaling (using Bray-Curtis similarity scores) on fecal microbiome samples; $n=11-12$. e Operational taxonomic units (OTUs) differentially expressed between Chow, Cycle, and Cafeteria groups. Data are expressed using both DeSeq2 (log(Fold change), FDRadjusted $p$-value $<0.05$ ), and LefSe (LDA score $>2$, FDR-adjusted $p<0.05$ ) scores. The color of the circle/bar denotes the group the OTU is enriched in (open: Chow, gray: Cycle, black: Cafeteria); $n=11-12 . \mathbf{f}$ Ether lipid metabolism, $\mathbf{g}$ flavone and flavonol biosynthesis, and $\mathbf{h}$ flavonoid biosynthesis predicted using PICRUSt from fecal microbiome data at 7 weeks. Data are expressed as box-and-whisker plots (min, IQR, max); $n=11-12$; data were analyzed by one-way ANOVA (FDR-adjusted overall $p$-value to account for multiple relevant pathways included in the analysis) followed by Tukeyadjusted post-hoc comparisons ( ${ }^{\mathrm{a}} p<0.05$ relative to Chow, ${ }^{\mathrm{b}} p<0.05$ relative to Cycle).

Table 2 Correlations between global microbiome composition and variables of interest.

\begin{tabular}{lllllll}
\hline Variable & SS & \multicolumn{1}{l}{ Pseudo-F } & P-value & $\mathbf{R}^{\mathbf{2}}$ & Residual DF \\
\hline Fat mass & 4061.7 & 2.716 & $\mathbf{0 . 0 0 4}$ & 0.0761 & 33 \\
Blood glucose & 3679.1 & 2.442 & $\mathbf{0 . 0 0 3}$ & 0.0689 & 33 \\
Leptin & 4818.1 & 3.272 & $\mathbf{0 . 0 0 2}$ & 0.0902 & 33 \\
Insulin & 3037.7 & 1.990 & $\mathbf{0 . 0 2 2}$ & 0.0569 & 33 \\
Plasma triglycerides & 3305.9 & 2.178 & $\mathbf{0 . 0 1 5}$ & 0.0619 & 33 \\
Liver weight & 4201.2 & 2.818 & $\mathbf{0 . 0 0 2}$ & 0.0787 & 33 \\
Heart weight & 1970.1 & 1.264 & 0.175 & 0.0369 & 33 \\
Average oocytes & 2539.5 & 1.648 & 0.056 & 0.0476 & 33 \\
per female & & & & & \\
Hippocampal Aif1 & 1875.6 & 1.176 & 0.287 & 0.0377 & 30 \\
Hippocampal II1b & 2711.6 & 1.730 & $\mathbf{0 . 0 5 0}$ & 0.0545 & 30 \\
Hippocampal II6 & 2394.6 & 1.518 & 0.099 & 0.0482 & 30 \\
Hippocampal Ikbkb & 2958.8 & 1.898 & $\mathbf{0 . 0 3 3}$ & 0.0595 & 30 \\
Hippocampal T/r4 & 1774.8 & 1.110 & 0.335 & 0.0357 & 30 \\
Hippocampal Glut1 & 2120.9 & 1.337 & 0.178 & 0.0427 & 30 \\
Hippocampal CIn5 & 1896.3 & 1.189 & 0.302 & 0.0381 & 30 \\
Hippocampal Syn1 & 2316.7 & 1.466 & 0.120 & 0.0466 & 30 \\
Place exploration ratio & 2895.4 & 1.892 & $\mathbf{0 . 0 2 2}$ & 0.0542 & 33 \\
\hline
\end{tabular}

Marginal distance-based linear modeling (dbLM) was performed using a Bray-Curtis similarity matrix of the global microbiome composition at the operational taxonomic unit level with variables of interest. Marginal dbLM interrogates the unique contribution of each predictor variable to the variance explained in the Bray-Curtis similarity matrix Pseudo-F and $p$-values were obtained using 999 permutations and bold values indicate $p$-values $\leq 0.05 ; N=$ $32-36$. All variable were tested, only those with a $p$-value $<0.4$ are presented. Aif1 allograft inflammatory factor $1, C \ln 5$ Claudin 5 , Glut1 glucose transporter 1 , $l k b k b$ inhibitor of nuclear factor $\mathrm{KB}$ kinase subunit- $\beta, \| 16$ interleukin- $1 \beta$, $/ 16$ interleukin-6, Syn1 synapsin 1, Trr4 Toll-like receptor 4.

phenotype when multiple anthropometric and microbiome predictors of NPR performance were considered, most likely due to issues of multicollinearity. These findings indicate that although increased adiposity and hippocampal gene expression may contribute to cognitive impairments observed in obesity ${ }^{46-48}$, these changes are highly related and are therefore difficult to disentangle.
Overall, it appears that different schedules of cafeteria diet induce incremental increases in hippocampal dysfunction and inflammation, and metabolic impairment, and it may be that some or all these changes must reach a threshold before diet-induced cognitive impairment is observed.

Cycle rats exhibited an intermediate microbiome composition that was significantly different from both Chow and Cafeteria groups, although exhibiting significant overlap with the Cafeteria group. This is similar to previous observations in mice exposed to 4-week cycles between chow and high-fat $\operatorname{diet}^{49}$ or chronic caloric restriction while on high-fat diet ${ }^{50}$, as well as our previous work with male rats exposed to longterm cafeteria diet cycling ${ }^{15}$. Although overall microbiome composition was altered, diet cycling protected female rats from the reductions in measures of $\alpha$-diversity typically seen with extended exposure to a cafeteria diet ${ }^{15,51}$. Reduced $\alpha$ diversity measures have been consistently reported in other diet-induced obesity models ${ }^{52,53}$, and following 4 -week periods of cycling between healthy and high-fat diets ${ }^{49}$. Our previous diet cycling work in male rats showed comparable reductions in richness between rats exposed to cycling and continuous cafeteria diet ${ }^{15}$, although this may have been due to either longer diet exposure of 16 weeks.

Naso-anal length and Coprobacter_OTU66 were identified as significant predictors of place task performance through multiple regression modeling. Naso-anal length is typically increased with cafeteria diet in rodents ${ }^{17,54}$ and, although it was not significantly increased with diet in this study, it was highly correlated with measures of adiposity, which is known to be negatively associated with cognition in both humans ${ }^{55,56}$ and rodents ${ }^{57}$. This relationship between spatial recognition and naso-anal length is likely specific to rodents, who continue to grow linearly across their lifespan. When naso-anal length was removed from the regression analysis, lean mass became a significant predictor of place task performance. Lean mass is also increased with an energy dense diet in rodents, most likely due to diet-induced growth as well as increased organ weights. Contrastingly, lean mass is protective against cognitive in aging humans ${ }^{58,59}$ and is positively associated with hippocampal-dependent lure discrimination in healthy young adults ${ }^{60}$. 
A)

\begin{tabular}{lllll}
\hline Variables & Coefficient & SEM & T-value & P-value \\
\hline Intercept & 2.592 & 0.554 & 4.681 & $<0.001$ \\
Naso-anal Length & -0.083 & 0.024 & -3.469 & 0.002 \\
Coprobacter_OTU66 & -0.002 & 0.001 & -3.624 & 0.001 \\
\hline
\end{tabular}

B)

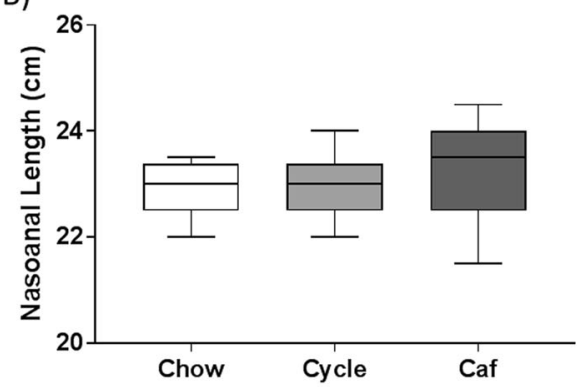

D)

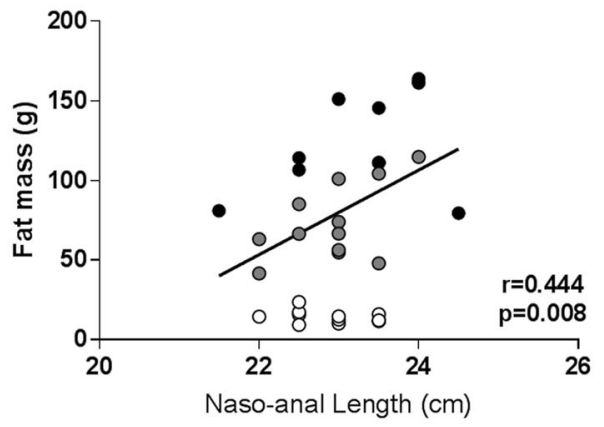

F)

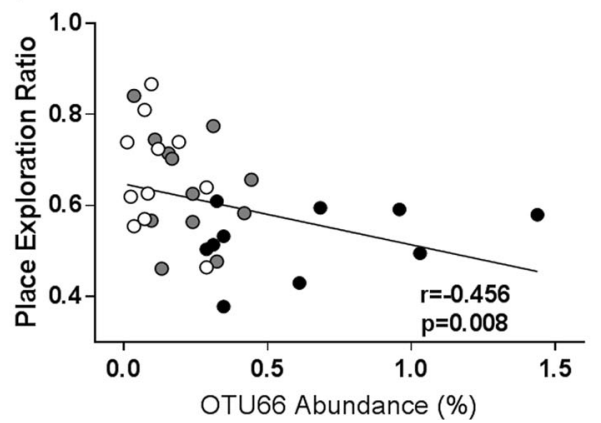

C)

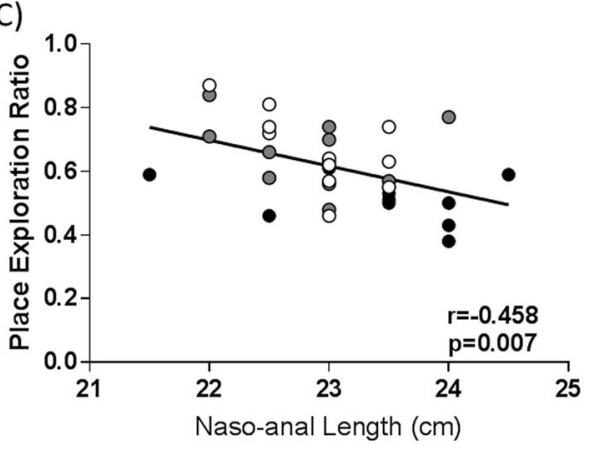

E)

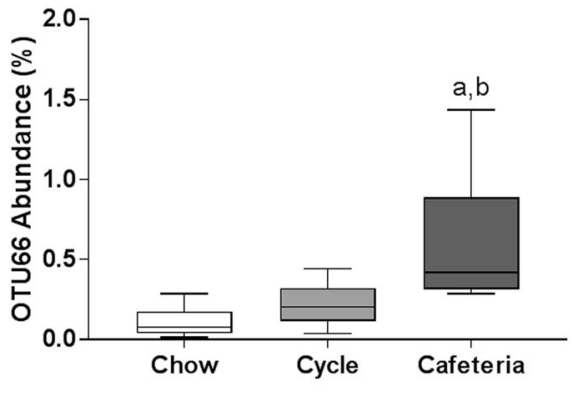

G)

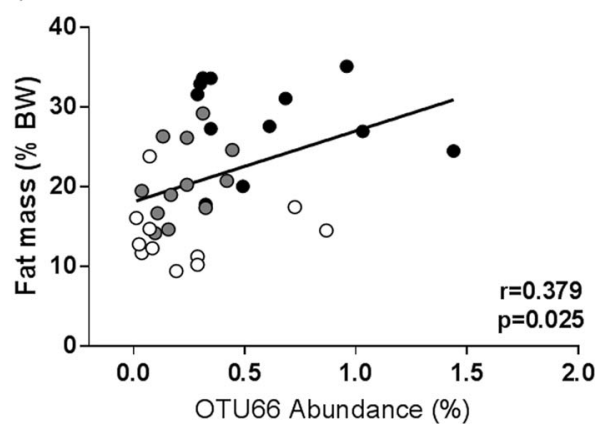

Fig. 3 Naso-anal length and Coprobacter_OTU66 are significant predictors of place task performance in a multiple regression model. a Final multiple regression model $(F(2,30)=12.28, p<0.001)$ predicting $41.3 \%$ of the variance in place task performance following the removal of nonsignificant predictors. b Naso-anal length at 7 weeks and its associations with (c) spatial recognition memory and (d) fat mass. e Relative abundance of Coprobacter_OTU66 at 7 weeks and its associations with (f) spatial recognition memory and (g) fat mass. Group data are expressed as box-and-whisker plots (min, IQR, max); $n=11-12$; data were analyzed by one-way ANOVA followed by Tukey-adjusted post-hoc comparisons ( ${ }^{a} p<0.05$ relative to Chow, ${ }^{b} p<0.005$ relative to (ycle). Associational data are expressed as scatterplots of individual values; $n=9-12$; data were analyzed by Pearson's correlations.

Coprobacter_OTU66 was putatively identified as $M$. intestinale strain YL27 and has only been recently sequenced ${ }^{61}$. In mice, this strain has been associated with a healthy phenotype ${ }^{62}$ and low-fat diet consumption ${ }^{61}$. Future studies manipulating this strain in rats may provide further insight into its role in diet-induced obesity and cognitive dysfunction.
It is important to note that we failed to detect any association between estrous stage and spatial task performance, but did observe a lengthening of proestrous and a reduction in diestrous stage $1 / 2$ of the Cafeteria rats, in line with other rodent work $^{21,63}$. We subsequently investigated the rats' ovaries and found that oocyte number was reduced by continuous cafeteria diet 
exposure. Similarly, others have shown that $\mathrm{Ob} / \mathrm{ob}$ mice exhibit reduced oocyte number ${ }^{64}$ and high-fat fed mice are less fertile due to increased oxidative stress-induced apoptosis and impaired oocyte maturation ${ }^{65}$.

In summary, our model of intermittent access to cafeteria diet identified OTU66_Coprobacter (high similarity to $M$. intestinale strain YL27) and naso-anal length, an indirect measure of adiposity and diet-induced growth, as predictive of differences in spatial recognition memory observed with differing schedules of cafeteria diet access in female rats. This approach appears useful for identifying microbiome composition differences that are relevant to phenotypic differences, rather than overall diet effects, and may prove useful in future studies investigating microbiome differences associated with cafeteria diet exposure.

\section{Acknowledgements}

The work was supported by an NHMRC project grant (\#1126929) to M.J.M. and R.F.W. We thank Robyn Lawler at the Biological Resources Centre, UNSW Sydney, for assistance with animal husbandry, Dr Michael Kendig and Josephine Yu for assistance with dissection, and Dr Kirsty Walters for estrous monitoring training. This research includes data processed by the computational cluster Katana supported by Research Technology Services at UNSW Sydney.

\section{Author details}

${ }^{1}$ School of Medical Sciences, UNSW, Sydney, NSW 2052, Australia. ${ }^{2}$ Fertility and Research Centre, School of Women's and Children's Health, UNSW, Sydney, NSW 2052, Australia. ${ }^{3}$ School of Psychology, UNSW, Sydney, NSW 2052, Australia

\section{Data availability}

The study metadata and sequence data are available in the European Nucleotide Archive under accession number PRJEB32323. All other data will be made available upon from the corresponding author on reasonable request.

\section{Conflict of interest}

The authors declare that they have no conflict of interest.

\section{Publisher's note}

Springer Nature remains neutral with regard to jurisdictional claims in published maps and institutional affiliations.

Supplementary Information accompanies this paper at (https://doi.org/ 10.1038/s41398-020-0734-9).

Received: 18 November 2019 Revised: 20 November 2019 Accepted: 27 November 2019

Published online: 27 January 2020

\section{References}

1. O'Brien, P. D., Hinder, L. M., Callaghan, B. C. \& Feldman, E. L. Neurological consequences of obesity. Lancet Neurol. 16, 465-477 (2017).

2. Beilharz, J. E., Maniam, J. \& Morris, M. J. Short-term exposure to a diet high in fat and sugar, or liquid sugar, selectively impairs hippocampal-dependent memory, with differential impacts on inflammation. Behav. Brain Res. 306, 1-7 (2016).

3. Kanoski, S. E., Zhang, Y., Zheng, W. \& Davidson, T. L. The effects of a highenergy diet on hippocampal function and blood-brain barrier integrity in the rat. J. Alzheimers Dis. 21, 207-219 (2010).

4. South, T., Westbrook, F. \& Morris, M. J. Neurological and stress related effects of shifting obese rats from a palatable diet to chow and lean rats from chow to a palatable diet. Physiol. Behav. 105, 1052-1057 (2012).
5. Gomez-Smith, M. et al. A physiological characterization of the Cafeteria diet model of metabolic syndrome in the rat. Physiol. Behav. 167, 382-391 (2016).

6. Higa, T. S., Spinola, A. V., Fonseca-Alaniz, M. H. \& Evangelista, F. S. Comparison between cafeteria and high-fat diets in the induction of metabolic dysfunction in mice. Int J. Physiol. Pathophysiol. Pharm. 6, 47-54 (2014).

7. Beilharz, J. E., Maniam, J. \& Morris, M. J. Short exposure to a diet rich in both fat and sugar or sugar alone impairs place, but not object recognition memory in rats. Brain Behav. Immun. 37, 134-141 (2014).

8. Lewis, A. R., Singh, S. \& Youssef, F. F. Cafeteria-diet induced obesity results in impaired cognitive functioning in a rodent model. Heliyon 5, e01412 (2019).

9. Ferreira, A., Castro, J. P., Andrade, J. P., Dulce Madeira, M. \& Cardoso, A. Cafeteria-diet effects on cognitive functions, anxiety, fear response and neurogenesis in the juvenile rat. Neurobiol. Learn Mem. 155, 197-207 (2018).

10. Beilharz, J. E., Kaakoush, N. O., Maniam, J. \& Morris, M. J. The effect of short-term exposure to energy-matched diets enriched in fat or sugar on memory, gut microbiota and markers of brain inflammation and plasticity. Brain Behav. Immun. 57, 304-313 (2016).

11. Hargrave, S. L., Davidson, T. L., Zheng, W. \& Kinzig, K. P. Western diets induce blood-brain barrier leakage and alter spatial strategies in rats. Behav. Neurosci. 130, 123-135 (2016).

12. Stranahan, A. M. et al. Diet-induced insulin resistance impairs hippocampal synaptic plasticity and cognition in middle-aged rats. Hippocampus $\mathbf{1 8}$ 1085-1088 (2008).

13. Beilharz, J. E., Kaakoush, N. O., Maniam, J. \& Morris, M. J. Cafeteria diet and probiotic therapy: cross talk among memory, neuroplasticity, serotonin receptors and gut microbiota in the rat. Mol. Psychol. 23, 351-361 (2018).

14. Del Bas, J. M. et al. Alterations in gut microbiota associated with a cafeteria diet and the physiological consequences in the host. Int. J. Obes. (Lond.) 42, 746-754 (2018).

15. Kaakoush, N. O. et al. Alternating or continuous exposure to cafeteria diet leads to similar shifts in gut microbiota compared to chow diet. Mol. Nutr. Food Res. 61, 1500815 (2017).

16. Martire, S. I., Westbrook, R. F. \& Morris, M. J. Effects of long-term cycling between palatable cafeteria diet and regular chow on intake, eating patterns, and response to saccharin and sucrose. Physiol. Behav. 139, 80-88 (2015).

17. Kendig, M. D., Westbrook, R. F. \& Morris, M. J. Patterns of access to cafeteriastyle diet determines fat mass and degree of spatial memory impairments in rats. Sci. Rep. 9, 13516 (2019).

18. George, V. A. \& Johnson, P. Weight loss behaviors and smoking in college students of diverse ethnicity. Am. J. Health Behav. 25, 115-124 (2001).

19. Paeratakul, S., York-Crowe, E. E., Williamson, D. A., Ryan, D. H. \& Bray, G. A. Americans on diet: results from the 1994-1996 Continuing Survey of Food Intakes by Individuals. J. Am. Diet. Assoc. 102, 1247-1251 (2002).

20. Leigh, S. J., Kendig, M. D. \& Morris, M. J. Palatable western-style cafeteria diet as a reliable method for modeling diet-induced obesity in rodents. JOVE 153, e60262 (2019).

21. Balasubramanian, P. et al. High fat diet affects reproductive functions in female diet-induced obese and dietary resistant rats. J. Neuroendocrinol. 24, 748-755 (2012).

22. Leigh, A. J., Stock, M. J., Lacey, J. H. \& Wilson, C. A. Diet-induced loss of cyclic ovarian function at normal body weight in a rodent model for bulimia nervosa. J. Reprod. Fertil. 112, 217-223 (1998).

23. Snider, A. P. \& Wood, J. R. Obesity induces ovarian inflammation and reduces oocyte quality. Reproduction (Camb., Engl.) 158, R79-R90 (2019).

24. Abbott, K. N., Morris, M. J., Westbrook, R. F. \& Reichelt, A. C. Sex-specific effects of daily exposure to sucrose on spatial memory performance in male and female rats, and implications for estrous cycle stage. Physiol. Behav. 162, 52-60 (2016).

25. Sutcliffe, J. S., Marshall, K. M. \& Neill, J. C. Influence of gender on working and spatial memory in the novel object recognition task in the rat. Behav. Brain Res. 177, 117-125 (2007).

26. Cora, M. C., Kooistra, L. \& Travlos, G. Vaginal cytology of the laboratory rat and mouse: review and criteria for the staging of the estrous cycle using stained vaginal smears. Toxicol. Pathol. 43, 776-793 (2015).

27. Andersen, C. L., Jensen, J. L. \& Orntoft, T. F. Normalization of real-time quantitative reverse transcription-PCR data: a model-based variance estimation approach to identify genes suited for normalization, applied to bladder and colon cancer data sets. Cancer Res. 64, 5245-5250 (2004).

28. Livak, K. J. \& Schmittgen, T. D. Analysis of relative gene expression data using real-time quantitative PCR and the 2(-delta delta $C(T)$ ) method. Methods (San Diego, CA) 25, 402-408 (2001). 
29. Schloss, P. D. et al. Introducing mothur: open-source, platform-independent, community-supported software for describing and comparing microbial communities. Appl. Environ. Microbiol. 75, 7537-7541 (2009).

30. Kozich, J. J., Westcott, S. L., Baxter, N. T., Highlander, S. K. \& Schloss, P. D. Development of a dual-index sequencing strategy and curation pipeline for analyzing amplicon sequence data on the MiSeq Illumina sequencing platform. Appl. Environ. Microbiol. 79, 5112-5120 (2013).

31. Zakrzewski, M. et al. Calypso: a user-friendly web-server for mining and visualizing microbiome-environment interactions. Bioinformatics (Oxf., Engl.) 33, 782-783 (2017).

32. McMurdie, P. J. \& Holmes, S. phyloseq: an R package for reproducible interactive analysis and graphics of microbiome census data. PLOS ONE 8, e61217 (2013).

33. Love, M. I., Huber, W. \& Anders, S. Moderated estimation of fold change and dispersion for RNA-seq data with DESeq2. Genome Biol. 15, 550 (2014).

34. Pruesse, E., Peplies, J. \& Glöckner, F. O. SINA: accurate high-throughput multiple sequence alignment of ribosomal RNA genes. Bioinformatics 28, 1823-1829 (2012).

35. Clarke, K. R. Non-parametric multivariate analyses of changes in community structure. Aust. J. Ecol. 18, 117-143 (1993).

36. Douglas, G. M. et al. PICRUSt2: an improved and extensible approach for metagenome inference. bioRxiv 672295 (2019).

37. Martire, S. I., Maniam, J., South, T., Holmes, N., Westbrook, R. F. \& Morris, M. J. Extended exposure to a palatable cafeteria diet alters gene expression in brain regions implicated in reward, and withdrawal from this diet alters gene expression in brain regions associated with stress. Behav. Brain Res. 265 132-141 (2014).

38. Barker, G. R., Bird, F., Alexander, V. \& Warburton, E. C. Recognition memory for objects, place, and temporal order: a disconnection analysis of the role of the medial prefrontal cortex and perirhinal cortex. J. Neurosci. 27, 2948-2957 (2007).

39. Barker, G. R. \& Warburton, E. C. When is the hippocampus involved in recognition memory? J. Neurosci. 31, 10721-10731 (2011).

40. Wen, L. et al. Innate immunity and intestinal microbiota in the development of Type 1 diabetes. Nature 455, 1109 (2008).

41. Reichelt, A. C., Morris, M. J. \& Westbrook, R. F. Daily access to sucrose impairs aspects of spatial memory tasks reliant on pattern separation and neural proliferation in rats. Learn Mem. 23, 386-390 (2016).

42. Kim, $\mathrm{H}$. et al. Caloric restriction improves diabetes-induced cognitive deficits by attenuating neurogranin-associated calcium signaling in high-fat diet-fed mice. J. Cereb. Blood Flow. Metab. 36, 1098-1110 (2016).

43. Li, L., Wang, Z. \& Zuo, Z. Chronic intermittent fasting improves cognitive functions and brain structures in mice. PLOS ONE 8, e66069 (2013).

44. Belegri, E., Eggels, L., Unmehopa, U. A., Mul, J. D., Boelen, A. \& la Fleur, S. E. The effects of overnight nutrient intake on hypothalamic inflammation in a free-choice diet-induced obesity rat model. Appetite $\mathbf{1 2 0}$ 527-535 (2018).

45. Almeida-Suhett, C. P., Graham, A., Chen, Y. \& Deuster, P. Behavioral changes in male mice fed a high-fat diet are associated with $I L-1 \beta$ expression in specific brain regions. Physiol. Behav. 169, 130-140 (2017).

46. Bove, R. M., Gerweck, A. V., Mancuso, S. M., Bredella, M. A., Sherman, J. C. \& Miller, K. K. Association between adiposity and cognitive function in young men: Hormonal mechanisms. Obesity (Silver Spring) 24, 954-961 (2016).
47. Gonzales, M. M. et al. Central adiposity and the functional magnetic resonance imaging response to cognitive challenge. Int. J. Obes. 38, 1193-1199 (2014).

48. Spyridaki, E. C. et al. The association between obesity and fluid intelligence impairment is mediated by chronic low-grade inflammation. Br. J. Nutr. 112 1724-1734 (2014)

49. Thaiss, C. A. et al. Persistent microbiome alterations modulate the rate of postdieting weight regain. Nature 540, 544-551 (2016).

50. Ravussin, Y. et al. Responses of gut microbiota to diet composition and weight loss in lean and obese mice. Obesity (Silver Spring) 20, 738-747 (2012).

51. Bhagavata Srinivasan, S. P., Raipuria, M., Bahari, H., Kaakoush, N. O. \& Morris, M. J. Impacts of diet and exercise on maternal gut microbiota are transferred to offspring. Front. Endocrinol. 9, 716-716 (2018).

52. Cotillard, A. et al. Dietary intervention impact on gut microbial gene richness Nature 500, 585 (2013).

53. Turnbaugh, P. J., Bäckhed, F., Fulton, L. \& Gordon, J. I. Diet-induced obesity is linked to marked but reversible alterations in the mouse distal gut microbiome. Cell Host Microbe 3, 213-223 (2008).

54. Shiraev, T., Chen, H. \& Morris, M. J. Differential effects of restricted versus unlimited high-fat feeding in rats on fat mass, plasma hormones and brain appetite regulators. J. Neuroendocrinol. 21, 602-609 (2009).

55. Papachristou, E. et al. The relationships between body composition characteristics and cognitive functioning in a population-based sample of older British men. BMC Geriatr. 15, 172 (2015).

56. Chen, J.-M. et al. Cognitive impairment among elderly individuals in Shanghai Suburb, China: association of C-reactive protein and its interactions with other relevant factors. Am. J. Alzheimers Dis. Other Dem. 29, 712-717 (2014).

57. Erion, J. R. et al. Obesity elicits interleukin 1-mediated deficits in hippocampal synaptic plasticity. J. Neurosci. 34, 2618-2631 (2014).

58. Noh, H. M. et al. Relationships between cognitive function and body composition among community-dwelling older adults: a cross-sectional study. BMC Geriatr. 17, 259 (2017).

59. Narimani, M., Esmaeilzadeh, S., Azevedo, L. B., Moradi, A., Heidari, B., KashfiMoghadam, M. Association between weight status and executive function in young adults. Medicina (Kaunas) 55, 363 (2019).

60. Golden, R. et al. Lean body mass, but not fat mass, is associated with hippocampal memory performance. Curr. Dev. Nutr. 3, P14-011-19 (2019).

61. Lagkouvardos, I. et al. The Mouse Intestinal Bacterial Collection (miBC) provides host-specific insight into cultured diversity and functional potential of the gut microbiota. Nat. Microbiol. 1, 16131 (2016).

62. Dobranowski, P. A., Tang, C., Sauvé, J. P., Menzies, S. C., Sly, L. M. Compositional changes to the ileal microbiome precede the onset of spontaneous ileitis in SHIP deficient mice. Gut Microbes 10, 578-598 (2019).

63. Chakraborty, T. R., Donthireddy, L., Adhikary, D. \& Chakraborty, S. Long-term high fat diet has a profound effect on body weight, hormone levels, and estrous cycle in mice. Med. Sci. Monit. 22, 1601-1608 (2016).

64. Cabello, E. et al. Effects of resveratrol on ovarian response to controlled ovarian hyperstimulation in ob/ob mice. Fertil. Steril. 103, 570-579.e571 (2015).

65. Hou, Y. J., Zhu, C. C., Duan, X., Liu, H. L., Wang, Q. \& Sun, S. C. Both diet and gene mutation induced obesity affect oocyte quality in mice. Sci. Rep. 6, 18858 (2016). 